\title{
A Distributed Core Network Architecture for 5G Systems and Beyond*
}

\author{
Shreyasee Mukherjee \\ WINLAB, Rutgers University, NJ \\ shreya@winlab.rutgers.edu
}

\author{
Ravishankar Ravindran \\ Huawei Research Center, CA \\ ravi.ravindran@huawei.com
}

\author{
Dipankar Raychaudhuri \\ WINLAB, Rutgers University, NJ \\ ray@winlab.rutgers.edu
}

\begin{abstract}
This paper presents a distributed core network architecture for use in future cellular systems. The proposed architecture addresses the performance bottlenecks and latency associated with the centralized control and data gateways used in today's mobile core networks. A fully distributed architecture for the mobile core is realized through the use of identifier-based protocol extensions to IP which run on base stations (eNodeB) and routers without the need for centralized gateways. The resulting "flat" mobile core network is capable of supporting a variety of mobility and IoT services with significantly lower latency and improved throughput relative to current solutions. Specific data plane service examples including service chaining and local VOIP are given. The paper concludes with an evaluation of control and data-plane overhead for a large US-scale cellular network operator in the proposed architecture.
\end{abstract}

\section{ACM Reference Format:}

Shreyasee Mukherjee, Ravishankar Ravindran, and Dipankar Raychaudhuri. 2018. A Distributed Core Network Architecture for 5G Systems and Beyond. In NEAT'18: ACM SIGCOMM 2018 Workshop on Networking for Emerging Applications and Technologies, August 20, 2018, Budapest, Hungary. ACM, New York, NY, USA, 6 pages. https://doi.org/10.1145/3229574.3229583

\section{INTRODUCTION}

With emerging and demanding user requirements, next generation cellular networking is expected to serve several heterogeneous services for $5 \mathrm{G}$ [4], with broadband access speed of $1 \mathrm{Gbps}$ and ultra low latency of $1 \mathrm{~ms}$ to serve certain application classes. However, while capacity of wireless links has improved exponentially with every new generation of the 3GPP standard, the evolution of the core network architecture has remained comparatively modest. The heterogeneity in the types of devices communicating is changing as well, from smart-phones to vehicular and internet of things (IoT) communication [1]. In this paper, we highlight the bottlenecks in the hierarchical gateway based core network design of current cellular systems and argue for a more distributed anchor-less model similar to the Internet backbone, that can scale elegantly to the increasing

\footnotetext{
${ }^{*}$ Research supported under NSF Future Internet Architecture - Next Phase (FIA-NP) grant CNS-1345295

Permission to make digital or hard copies of all or part of this work for personal or classroom use is granted without fee provided that copies are not made or distributed for profit or commercial advantage and that copies bear this notice and the full citation on the first page. Copyrights for components of this work owned by others than ACM must be honored. Abstracting with credit is permitted. To copy otherwise, or republish, to post on servers or to redistribute to lists, requires prior specific permission and/or a fee. Request permissions from permissions@acm.org.

NEAT'18, August 20, 2018, Budapest, Hungary

(C) 2018 Association for Computing Machinery.

ACM ISBN 978-1-4503-5907-8/18/08 . .\$15.00

https://doi.org/10.1145/3229574.3229583
}

number and heterogeneity of current and future subscribers while also enabling new mobility and IoT services.

Today's cellular network architecture was designed to manage per-device connectivity at service, control and data plane level with fine-grained mobility and QoS management. There was a need to monetize the high spectrum cost and comply with regulatory requirements resulting in functions towards enforcing various policy, charging and satisfying legal intercept requirements. While this architectural model has survived over four generations of standardization upgrades, recent works have exposed the drawbacks and bottlenecks in its service and packet gateway based data forwarding $[13,14,17]$. There is an increasing diversity in the types of devices being manufactured to use cellular, a significant shift from smartphones and PCs. IoTs in industrial control and trucking requires low latency and reliability, simple sensing devices benefit from low overhead connection, minimum network-state and session-less communication. On the other hand, smartphones and vehicular scenarios demand faster and seamless handovers with increasing bitrates and mobility. Such heterogeneous requirements, coupled with the increasing need to provide in-network services such as content caching and edge-compute, require intelligent data plane forwarding mechanisms within the core network. The ongoing standardization efforts in $5 \mathrm{G}$, provides an opportunity to understand the shortcomings of the $4 \mathrm{G}$ network architecture and propose backward compatible solutions that can address the requirements of scale and heterogeneity for future cellular networks. The key contributions of this work are as follows:

- Enumerate the scalability bottlenecks in the evolved packet core (EPC) of cellular networks.

- Propose a distributed core network architecture for handling increased scale at significantly lower overhead, while preserving identity and security requirements of cellular networks and enabling flexible creation of new mobility and IoT services.

- Provide preliminary results from a large scale simulation study comparing the 4G EPC architecture with the flat network architecture in terms of scalability and control overhead.

\section{THE CELLULAR NETWORK}

The cellular network architecture was designed primarily for longlived data sessions for power and resource-sufficient user-equipments (UEs). Following the terminologies of the evolved packet core (EPC) in $4 \mathrm{G}$, this consists of a centralized mobility management entity (MME) with a home subscription server (HSS), and packet and service gateways (PGW and SGW). In this setup, every time an UE requires data connectivity, a list of handshakes and messaging between different components of the EPC and the RAN needs to take place before any application-level data packet can be transmitted. Fig. 1 highlights the control messages for uplink data network connectivity for an UE. The key functionalities include authenticating 


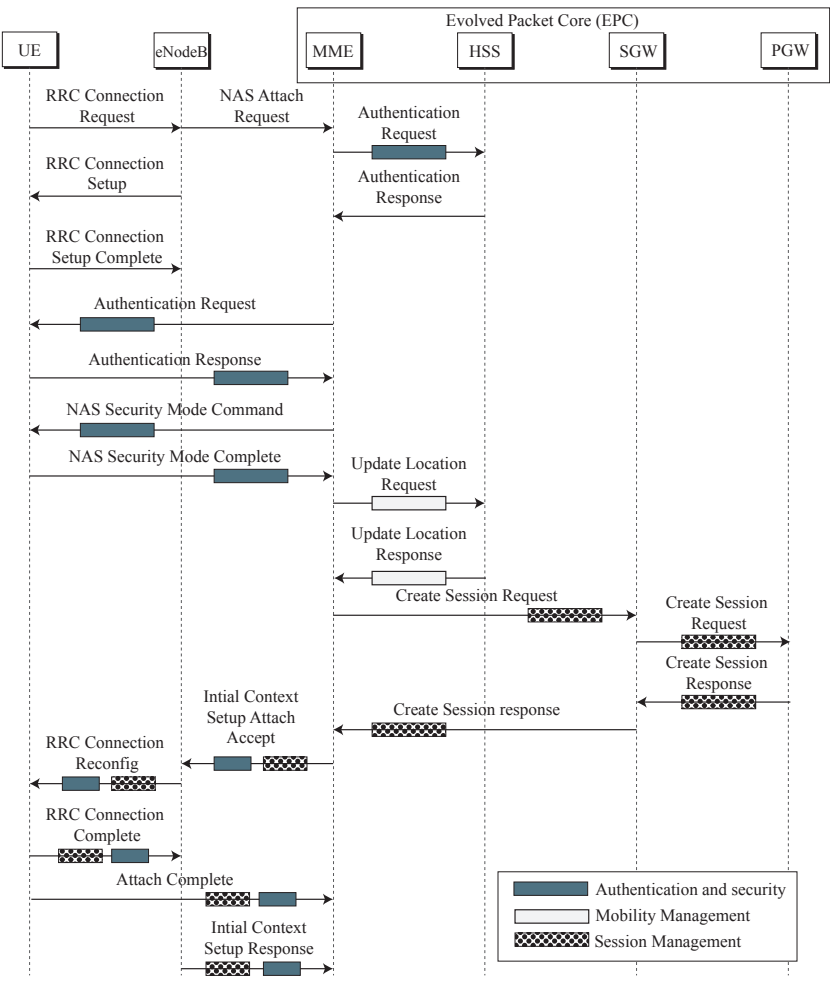

Figure 1: Control messaging to establish uplink data connectivity for a user equipment (UE) using $4 \mathrm{G}$

the UE, updating its location in terms of the basestation (eNB) it is currently connected to, and setting up dedicated bearers from the eNB to its serving gateway. Not only is this latency intensive, this procedure repeats every time a UE switches from idle to active state. As seen from Fig. 1, the total number of control messages can be as high as 17 for every idle to active transition.

The components in the EPC were designed to distribute functionality across different modules that can be independently upgraded or modified. The HSS is in charge of device-authentication and on-boarding. The MME handles mobility of the UE in the network and mediates the protocol exchanges between the eNBs and the rest of the core network. The service gateways maintain long-lived sessions per UE and allow all traffic to enter and exit through limited ingress/egress points in the network for effective enforcement of policies, admission control and charging.

One of the main shortcomings of this hierarchical architecture design is that the data-plane path is setup and managed between the eNB and the gateways using the GTP tunneling protocol [5]. The gateways perform this task, by setting up dedicated tunnels for each UE, identified by tunnel identifiers, generated during the protocol exchanges shown in Fig. 1. Every data packet then needs to be encapsulated and de-encapsulated at the tunnel endpoints (eNB and the PGW), which adds to the data overhead and complexity of the architecture design. These tunnels are torn down and re-established with every mobility event, creating even more overhead. To add to that, as the authors in [24] show, most US based cellular providers have only $4-6$ such gateways to handle

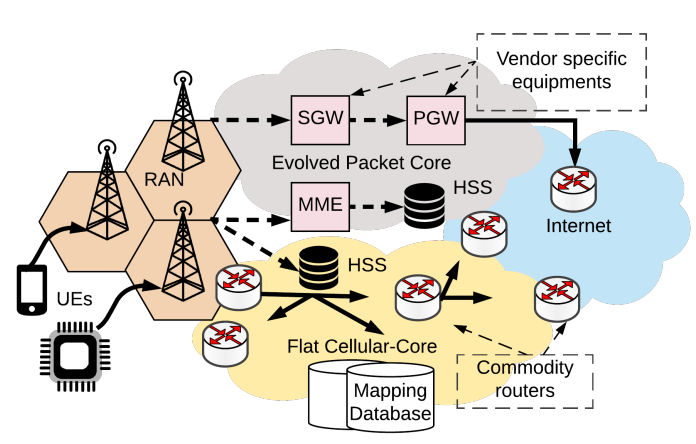

Figure 2: Anchorless distributed cellular core network with no gateways and vendor-specific equipments in contrast to the evolved packet core (EPC)

traffic from millions of subscribers. This still works if the number of devices connecting through the core is reasonable, they do not have stringent latency and control packet processing limitations, and, maintain long-lived connectivity patterns (active tunnels), similar to smartphones. However, once we start to relax some these assumptions for devices such as sensors in a smart-vehicle, or industrial control and interpolate the predicted growth of heterogeneous devices connecting through $5 \mathrm{G}$, the gateways become source of serious bottlenecks, as shown in our evaluations in Sec. 4.

\section{AN ANCHOR-LESS CELLULAR CORE}

In this section, we introduce the concept of a distributed, anchorless core network architecture for cellular networks, that scales elegantly with number and heterogeneity of devices. As shown in Fig. 2, the flat core network eliminates existing MME and gateways and instead utilizes commodity routers that are connected in a distributed manner, similar to the Internet backbone. The same routing functionality is also embedded into the base stations (eNodeBs). The core network, although flat, still needs to perform the basic functionalities of a cellular network, namely, authentication and device onboarding, mobility management and policy and charging. HSS still operates as a database for UE subscription management and to handle initial device onboarding. Architecturally, we propose two key changes compared to the EPC.

First, instead of the MME, we introduce a distributed mapping system that is not restricted to mapping end-devices to locations, but allows identifiers associated with devices, services and even context to be mapped into a set of locators. This concept of separation between identifiers and routable locations has been proposed by both the academic community $[8,18]$ and the standards bodies $[6,9]$, as a means to address mobility, multi-homing, service-management and migration, content and context-addressability and to allow a flexible mechanism to introduce new services into the network in the future. Introducing a similar mapping system within the cellular packet core will not only reduce bottleneck at the MMEs (which handle 3 times more control traffic than the gateways, as seen in Fig. 1), but also allow enhanced services such as multi-homing, multicast, efficient traffic engineering to be instantiated and managed using the same framework. Prior work on mapping system design and implementation has shown it to be scalable to billions of devices with query latencies lower than 10 milliseconds [7, 10, 11, 19, 23]. 


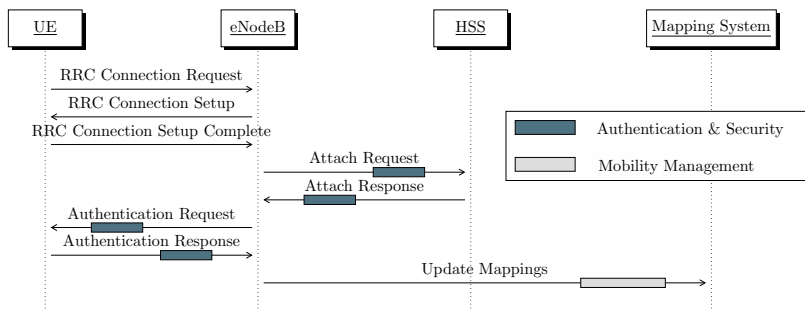

Figure 3: Authentication and device onboarding with a distributed mapping system and no gateways

Secondly, the flat core network has no gateways and this brings in several additional advantages to the design. (i) It reduces the control overhead and protocol exchange, every time an UE goes from idle-to-active stage; (ii) It removes the potential bottlenecks in the data-plane and allows for a more distributed routing of packets through the core into the Internet; and, (iii) It allows newer dataplane service to be instantiated easily which is not possible using GTP.

The device onboarding steps are shown in Fig. 3. Whereas, the RRC connection setup, authentication and UE attachment to the network remains unchanged, the eNBs now need to perform the policy and QoS enforcement, typically performed at the $\mathrm{P} / \mathrm{S}$ gateways when data is exiting or entering the core network through GTP tunnels. Distributing the charging functionality close to the edge reduces the control bottleneck at the limited number of gateways as well as allows for interesting charging policies to be implemented, such as charging local traffic differently than traffic traversing multiple hops out of the service provider network. In addition to that, the eNBs in this distributed identity based architecture need to update device ID to routable locator (eNB address) mapping in the mapping database, as shown.

Note that this architecture design is based on MobilityFirst [18], which is a future internet architecture with its own semantics of flat cryptographically secure identifiers and a distributed hash map based mapping system [10,23]. However, the cellular core network design proposed is agnostic to any naming, name-address separation protocols and mapping system designs and therefore, can work with any of the alternative name-based architectures that use IP semantics [6, 8, 9]. Using MobilityFirst as an example, Fig. 4 summarizes the protocol exchanges to establish data connectivity for an identity-enabled UE. As shown in Fig. 4(a), during UE attachment, policy and charging policies are made available to the eNB for that particular UE and a UE identifier to eNB address is inserted in the mapping database. When the eNB next receives a data packet from the UE, it can look up the appropriate QoS policies, query the database for the destination identifier to address mapping and forward the packet accordingly. The packet structure shown here, carries both the identifier and the routable address, following MobilityFirst packet syntax. For alternative identity-based architectures this packet format and the mapping-function at the eNBs would be different. For example, LISP [6] encapsulates the original packet with a new IP header carrying the destination IP address, while ILA rewrites the destination identifier with the destination address [9]. However, the core network architecture, the distributed mapping

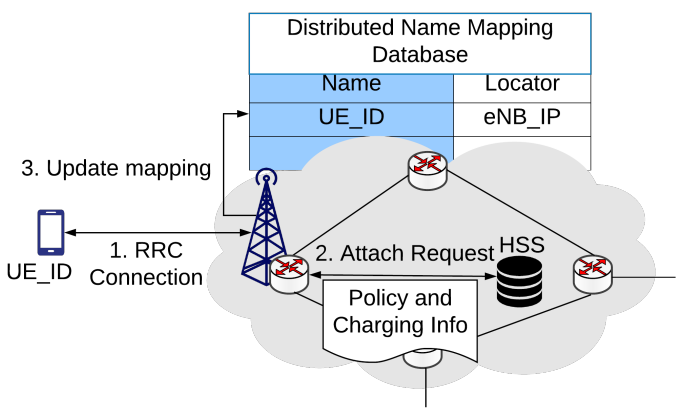

(a) Control plane steps

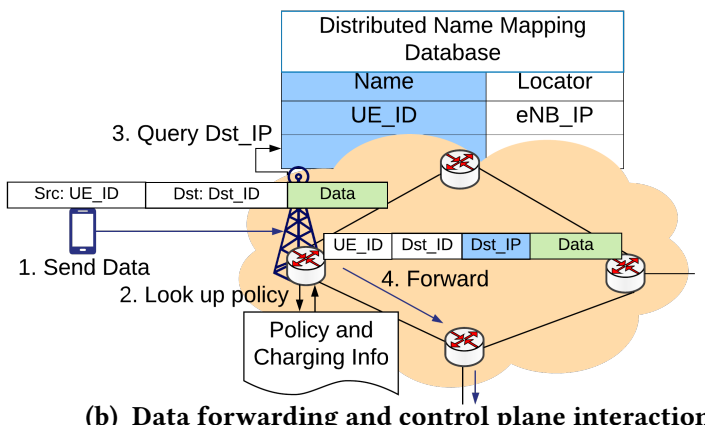

Figure 4: Protocol Exchanges using MobilityFirst

system and the protocol syntax can adapt well to such alternative designs.

Next, we walk through a few data-plane services to highlight the benefits of using the distributed core network.

\subsection{Data-plane services}

In this section, we walk through two data-plane services, namely, (i) a voice-over-IP call between two smartphones both subscribed to the same network carrier; and, (ii) service chaining and flexible policy enforcement at a cellular service provider. While both of them are realizable in a traditional cellular network, our goal is to highlight the benefits and flexibility that a distributed architecture provides.

VOIP between local mobile users: We start with the assumption that both the smartphones are in RRC connected state and have already authenticated and attached to the network following the protocol diagram in Fig. 3. As shown in Fig. 5(a), UE 1 is initiating a data flow to UE2. Following the concept of a identity-locator split architecture, the first packet at the basestation will incur a mapping database lookup to find the routable locator for UE2 (which in this case is the address of eNB2). Our design assumes that this mapping is cached at the basestation for the lifetime of this VOIP flow and hence, consequent packets would not require further lookups. Based on the core network topology and the routing protocols within the core network, the packets will be forwarded across the shortest path through the network to eNB2. If we contrast this to what would happen for the same application in the EPC, we will observe that all packets from eNB1 will be encapsulated and tunneled to a PGW deep into the network, from where they need to be de-encapsulated, followed by encapsulated a second time and 
sent via a second tunnel to eNB2. The actual route followed by the packet may be quite non-optimal, both from the user and the network perspective, especially considering the limited number of PGWs per carrier [24].

Service chaining: Next, we describe a second usecase, where a cellular service provider wants to implement flexible policies, QoS and network functions within the network. In this case, service functions are instantiated by assigning identities to the service and storing an up-to-date mapping between the service identifier and its location in the mapping system. As shown in Fig. 5(b), network functions such as filtering, and deep packet inspection (DPI) can be chained together for a particular flow, simply by inserting the correct order of mapping of locators in the mapping system. For example, based on the SLA agreements and QoS policies, data packets from UE 1 are first directed to a filtering service, from where a second lookup in the mapping system is made to direct it to a DPI service, after which it is forwarded to an egress router. On the other hand, packets from UE2, which could have a different agreement for lower latency, is forwarded out into the backbone through the closest egress router. Avoiding P/S gateways allows the network to implement many such flexible policies without having to instantiate and maintain multiple GTP tunnels between each network function. In addition, decoupling the service names from their routable addresses provides flexible traffic engineering capabilities to the network, as any function can now be easily moved or assigned to multiple locations in the network, simply by inserting the correct mappings in the mapping system.

\section{EVALUATION}

\subsection{Realistic System Model}

We focus on a single US based cellular carrier, referred henceforth as $C 1$, which reported 138.83 million wireless subscribers in 2017 [20]. We also consider future predictions of wireless subscriber growth [1] and IoTs using cellular. [22] predicts there will be as many as 52547 IoTs per cell site in the near future. Based on our calculation that would result in about 33 billion IoTs for $C 1$. We assume a more modest growth of 33 million in our simulation. We parse the opencellid dataset to obtain crowd-sourced data of about $650 \mathrm{~K}$ cell sector locations for $C 1$ [21]. Note that the actual number of eNBs will depend on the number of cell sectors of each of $C 1$ 's eNBs, the information of which was unfortunately not available. We assume that $C 1$ has at-most 6 PGWs (based on [24]), and then parametricize the number of SGWs and MMEs as multiples of PGW. For the data-plane topology, we parse reported router-level topology of $C 1$ from Caida [2], that results in a distributed topology of about $82 \mathrm{~K}$ nodes and $441 \mathrm{~K}$ links. The simulation parameters are summarized in Table 1.

\subsection{Control Overhead Analysis}

Based on the above topology, we simulate UE attach requests and analyze the control overhead incurred by the components in the EPC and in the mapping system of the proposed distributed cellular core network. As described in Sec. 2, the UE attachment procedure repeats every time an UE transitions from idle-to-active state. We refer to this as the RRC connection periodicity. To obtain reasonable numbers for this connection periodicity, we parse more than

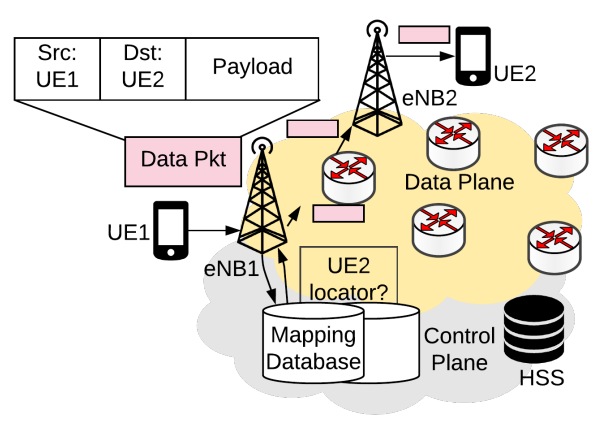

(a) VOIP call between two subscribers of the same network

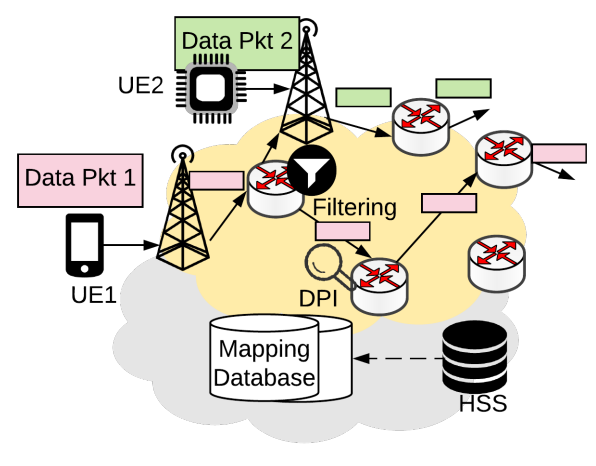

(b) Instantiating services and QoS policies in the core network

Figure 5: Different data-plane services enabled by the distributed core

Table 1: Simulation Parameters

\begin{tabular}{|l|l|}
\hline Parameter & Value \\
\hline UEs(smartphones) & $138.8310^{6}$ \\
\hline UEs(IoTs) & $3310^{6}$ \\
\hline eNB cell sites & 637494 \\
\hline PGWs & 6 \\
\hline SGWs & $\{6,12,18,24\}$ \\
\hline MMEs & $\{6,12,24,36,48\}$ \\
\hline Routers & 82756 \\
\hline Links & 441136 \\
\hline
\end{tabular}

2500 LTE logs collected by users over a period of months and available as part of the MobileInsight project [13]. For IoT devices, we synthetically generate RRC connection periodicity, following the guidelines in [22]. Fig. 6 plots the cumulative distribution function of the RRC sleep periodicity. The dotted lines ('All Carriers' and 'Synthetic IoT') are the ones fed into our simulator. As seen from the figure, while the real data from the smartphones show a lot of variations in the RRC sleep periodicity, the guidelines for IoT connectivity pattern only have 4 categories of sleep periodicity (wakeup once a day, once every 2 hours, once every hour, and once every 30 minutes). We assumed a 5\% standard deviation from these means when generating the synthetic data. 


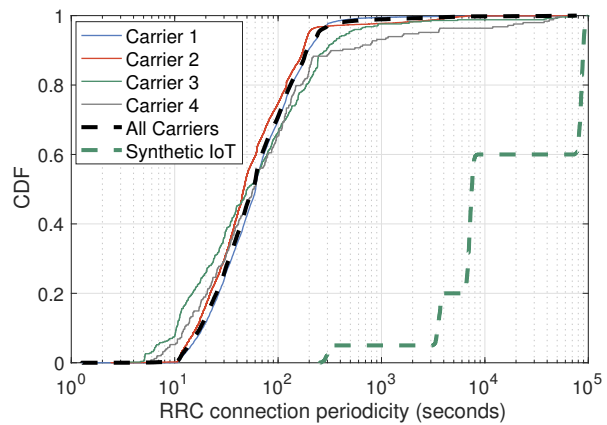

Figure 6: UE wakeup intervals for smartphones (real-world data) and IoTs (synthetic data)

Next, we simulated 138.83M UEs following the connectivity pattern from Fig. 6 and sending attach requests into the network. For the EPC, Fig. 7 plots the average and worst case control packet overhead at the PGW, SGW and the MME, based on the protocol exchanges explained in Sec. 2. Following Cisco 2016-2021 forecasts, we next increase the number of UEs in the simulation to predict average overheads for the year 2021 as shown. Finally, we add in 33M IoT devices to strain the network further. As seen from the figure, the MMEs are a key source of potential bottlenecks, with maximum load reaching over $5 \mathrm{M}$ packets/sec. As the MME is the mediator of setting up a data session from the eNB to the PGW, overloading the MME, will not only create latency bottlenecks, but will also lead to delays in mobility handover scenarios (not considered for this evaluation).

We next simulate the UE attachment protocols in the distributed core, and analyze the control overhead at the mapping database for the same set of UEs and control traffic model. The simulation implements a distributed hash table based mapping system, in which all the routers of the core network participate in. In summary, each router stores part of the logically centralized database and responds to updates and queries from the eNBs. For reliability and to lower lookup latencies, each mapping is stored in $k$ different locations $(k>1)$ across the network. Please refer to [23] for more details on the mapping system design. As seen from Fig. 8, the overhead at each of these mapping database is negligible, even with increasing values of $k$, compared to the the overhead at the MME in the EPC. This is due to two main reasons: (i) The protocol exchanges are simpler in the distributed core, and, (ii) the database is physically distributed across $82 \mathrm{~K}$ nodes in the network, resulting in reduced overhead per node. Note, that the assumption here is that commodity routers will be able to adequately handle slightly higher overhead and computation in maintaining these distributed databases, as has been shown in [10, 23].

\subsection{Data-plane Analysis}

In order to highlight the benefits of removing session gateways and GTP tunneling, we next evaluate the VOIP use-case described in Sec. 3.1, where two local subscribers are communicating over the core network. There are 6 PGWs in the topology which have been randomly chosen from the set of egress routers of $C 1$ (routers which have atleast one inter-domain link to a different autonomous
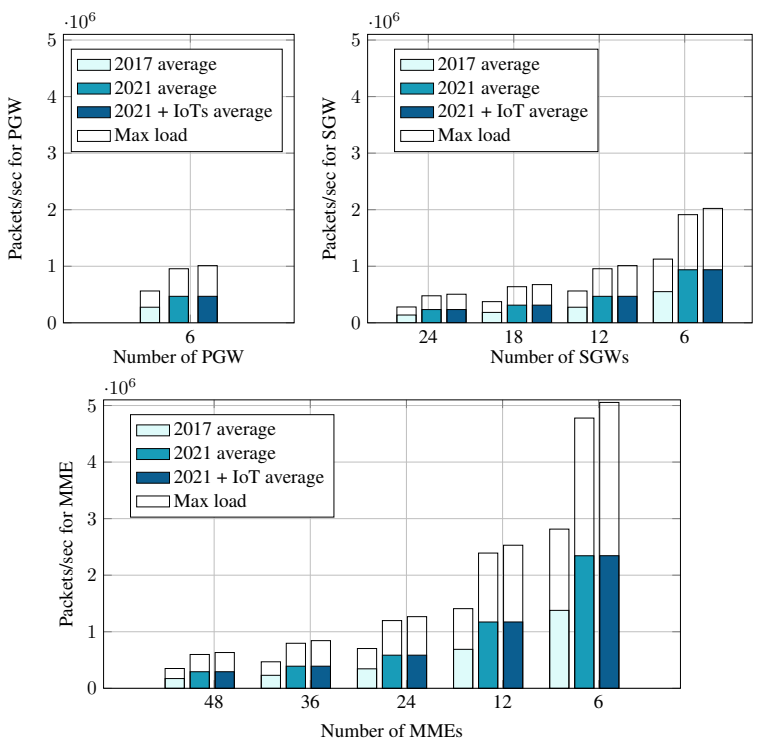

Figure 7: Control overhead at each PGW, SGW and MME for subscribers of an US-scale carrier for the year 2017 and forecasted increase for 2021 .

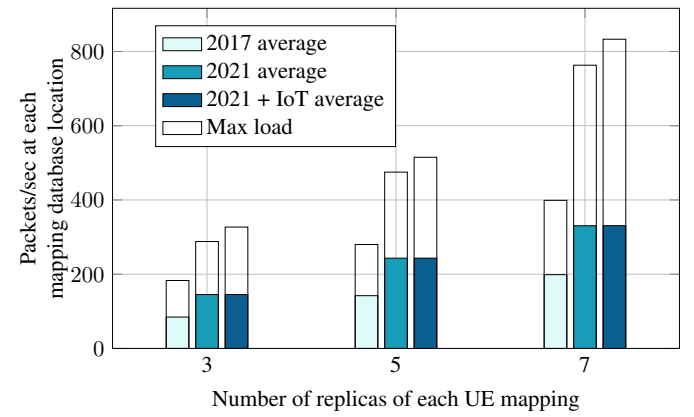

Figure 8: Control overhead at each of the locations of the distributed mapping system for the year 2017 and forecasted increase for 2021.

system in the Caida topology). We choose 10000 random pairs of UEs and assume that the QoS policies for both them allow shortest path forwarding in the core. As mentioned earlier, when using the EPC core, packets from UE1 are tunneled to the closest PGW, from where they are forwarded through a second tunnel to UE2. For the distributed core, packets are simply forwarded along the shortest path between UE1 and UE2. As shown in Fig. 9, the distributed forwarding for 10000 random pairs of UEs results in considerable improvement in packet hops for each flow, which could potentially reduce end-to-end latency and allow better traffic distribution in the network.

\section{RELATED WORK}

Works on the cellular core network architecture can be classified into three categories. First, few works have introduced SDN in 


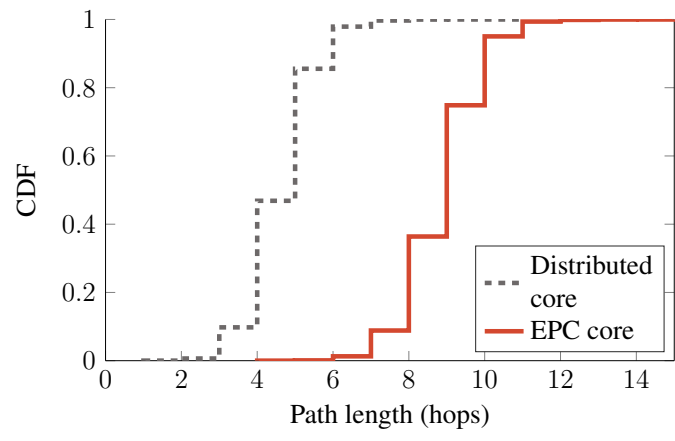

Figure 9: Data flow path length for 10,000 random pairs of UEs, both subscribed to the cellular network

the core network to allow for flexible traffic engineering and finegrained policies $[12,15]$. However, they assume the protocols exchanges and the MMEs and gateways remain unchanged. The second body of work involve virtualizing the EPC functionality $[3,16]$, such that a software implementation of the EPC can be instantiated and migrated at will by the service provider. While this is valuable and has promise to be used in $5 \mathrm{G}$ to allow multiple software cores to share the network resources, as shown through our evaluations, the EPC core components are themselves not scalable. Finally, recent proposals also look into smarter backward compatible techniques that tweak part of the protocols in order to reduce overall latency and overhead $[13,14,17]$. These are the ones most closely related to our work but are complimentary, as utilizing their NFV and virtualization techniques will provide additional latency reduction for our proposed architecture. There is also a separate body of work on enabling IoT and low power communication in cellular [22]. However, they do not focus on low latency scenarios and are specific to low bit rate communication, whereas, our proposed architecture is more generic and supports multiple types of devices.

\section{CONCLUSION}

This paper proposed a distributed core network architecture for $5 \mathrm{G}$ cellular systems. The proposed architecture removes traditional gateways from the cellular core and introduces a distributed mapping system for mobility management and flexible support for newer services and heterogeneous devices, such as IoTs. Our results from a large-scale simulation of a US-based cellular provider show significant reduction in control overhead and smaller data-plane path lengths. Ongoing work includes prototyping the proposed architecture on commodity hardware and USRP radio front-ends using Click software router and Open Air Interface. As part of our future work, we plan to present detailed experimental results on latency and mobility studies in the ORBIT radio testbed.

\section{REFERENCES}

[1] 2017. Cisco Visual Networking Index: Global Mobile Data Traffic Forecast Update, 2016-2021 White Paper. https://www.cisco.com/c/en/ us/solutions/collateral/service-provider/visual-networking-index-vni/ mobile-white-paper-c11-520862.html. (2017). Accessed: 2018-06-08.

[2] 2018. Center for Applied Internet Data Analysis. http://www.caida.org/. Accessed: 2018-06-08.

[3] Arsany Basta, Wolfgang Kellerer, Marco Hoffmann, Klaus Hoffmann, and ErnstDieter Schmidt. 2013. A virtual SDN-enabled LTE EPC architecture: A case study for S-/P-gateways functions. In Future Networks and Services (SDN4FNS), 2013 IEEE SDN for. IEEE, 1-7.

[4] Rachid El Hattachi and Javan Erfanian. 2015. Ngmn $5 \mathrm{~g}$ white paper, a deliverable by the ngmn alliance. https://www.ngmn.org/fileadmin/ngmn/content/ downloads/Technical/2015/NGMN_5G_White_Paper_V1_0.pdf. NGMN Alliance, February (2015). Accessed: 2018-06-08.

[5] TS ETSI. 2010. 129281 V9. 3.0 (2010-06) Technical Specification, GPRS Tunnelling Protocol for User Plane (GTPv1- U). Universal Mobile Telecommunications System (UMTS) (2010).

[6] Dino Farinacci, Darrel Lewis, David Meyer, and Vince Fuller. 2013. The locator/ID separation protocol (LISP). RFC 6830 (2013).

[7] V Fuller, D Farinacci, D Meyer, and D Lewis. 2013. Locator/ID separation protocol alternative logical topology (LISP+ ALT). RFC 6836 (2013).

[8] Andrei Gurtov, Miika Komu, and Robert Moskowitz. 2009. Host identity protocol: identifier/locator split for host mobility and multihoming. The Internet Protocol fournal 12, 1 (2009), 27-32.

[9] Tom Herbert and Petr Lapukhov. 2018. Identifier-locator addressing for IPv6. Internet Draft (2018).

[10] Y. Hu, R. D. Yates, and D. Raychaudhuri. 2015. A Hierarchically Aggregated In-Network Global Name Resolution Service for the Mobile Internet. In WINLAB TR 442.

[11] Amit Jain, Anton Smirnov, Darrel Lewis, Vina Ermagan, and Vince Fuller. 2017. Locator/ID Separation Protocol Delegated Database Tree (LISP-DDT). RFC 8111 (2017).

[12] Xin Jin, Li Erran Li, Laurent Vanbever, and Jennifer Rexford. 2013. Softcell: Scalable and flexible cellular core network architecture. In Proceedings of the ninth ACM conference on Emerging networking experiments and technologies. ACM, 163-174.

[13] Yuanjie Li, Chunyi Peng, Zengwen Yuan, Jiayao Li, Haotian Deng, and Tao Wang. 2016. Mobileinsight: Extracting and analyzing cellular network information on smartphones. In Proceedings of the 22nd Annual International Conference on Mobile Computing and Networking. ACM, 202-215.

[14] Ali Mohammadkhan, KK Ramakrishnan, Ashok Sunder Rajan, and Christian Maciocco. 2016. CleanG: A Clean-Slate EPC Architecture and ControlPlane Protocol for Next Generation Cellular Networks. In Proceedings of the 2016 ACM Workshop on Cloud-Assisted Networking. ACM, 31-36.

[15] Mehrdad Moradi, Wenfei Wu, Li Erran Li, and Zhuoqing Morley Mao. 2014. SoftMoW: Recursive and reconfigurable cellular WAN architecture. In Proceedings of the 10th ACM International on Conference on emerging Networking Experiments and Technologies. ACM, 377-390.

[16] Zafar Ayyub Oazi, Phani Krishna Penumarthi, Vyas Sekar, Vijay Gopalakrishnan, Kaustubh Joshi, and Samir R Das. 2016. KLEIN: A minimally disruptive design for an elastic cellular core. In Proceedings of the Symposium on SDN Research. ACM, 2.

[17] Zafar Ayyub Qazi, Melvin Walls, Aurojit Panda, Vyas Sekar, Sylvia Ratnasamy, and Scott Shenker. 2017. A High Performance Packet Core for Next Generation Cellular Networks. In Proceedings of the Conference of the ACM Special Interest Group on Data Communication. ACM, 348-361.

[18] Dipankar Raychaudhuri, Kiran Nagaraja, and Arun Venkataramani. 2012. Mobilityfirst: a robust and trustworthy mobility-centric architecture for the future internet. ACM SIGMOBILE Mobile Computing and Communications Review 16, 3 (2012), 2-13.

[19] Abhigyan Sharma, Xiaozheng Tie, Hardeep Uppal, Arun Venkataramani, David Westbrook, and Aditya Yadav. 2015. A global name service for a highly mobile internetwork. ACM SIGCOMM Computer Communication Review 44, 4 (2015), 247-258.

[20] statista. 2018. Wireless subscriptions market share by carrier in the U.S. from 1st quarter 2011 to 4 th quarter 2017. https://www.statista.com/statistics/199359/ market-share- of-wireless-carriers-in-the-us-by-subscriptions/. (2018). Accessed: 2018-06-08.

[21] unwiredlabs. 2018. The world's largest Open Database of Cell Towers. https: //www.opencellid.org/. (2018). Accessed: 2018-06-08.

[22] 3GPP TR 45.820 V13.1.0. 2015. cellular system support for ultra-low complexity and low throughput internet of things (CIoT). (2015).

[23] Tam Vu, Akash Baid, Yanyong Zhang, Thu D Nguyen, Junichiro Fukuyama, Richard P Martin, and Dipankar Raychaudhuri. 2012. Dmap: A shared hosting scheme for dynamic identifier to locator mappings in the global internet. In Distributed Computing Systems (ICDCS), 2012 IEEE 32nd International Conference on. IEEE, 698-707.

[24] Oiang Xu, Junxian Huang, Zhaoguang Wang, Feng Oian, Alexandre Gerber, and Zhuoqing Morley Mao. 2011. Cellular data network infrastructure characterization and implication on mobile content placement. In Proceedings of the ACM SIGMETRICS joint international conference on Measurement and modeling of computer systems. ACM, 317-328. 